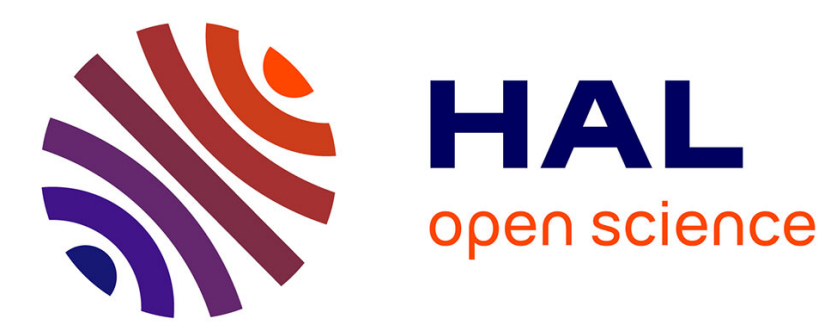

\title{
Hierarchical predictive control strategy of microalgae culture in a photobioreactor
}

Seif Eddine Benattia, Sihem Tebbani, Didier Dumur

\section{To cite this version:}

Seif Eddine Benattia, Sihem Tebbani, Didier Dumur. Hierarchical predictive control strategy of microalgae culture in a photobioreactor. 19th International Conference on System Theory, Control and Computing (ICSTCC), Oct 2015, Cheile Gradistei, Romania. 10.1109/ICSTCC.2015.7321298 . hal-01257011

\section{HAL Id: hal-01257011 \\ https://hal-centralesupelec.archives-ouvertes.fr/hal-01257011}

Submitted on 12 Mar 2020

HAL is a multi-disciplinary open access archive for the deposit and dissemination of scientific research documents, whether they are published or not. The documents may come from teaching and research institutions in France or abroad, or from public or private research centers.
L'archive ouverte pluridisciplinaire HAL, est destinée au dépôt et à la diffusion de documents scientifiques de niveau recherche, publiés ou non, émanant des établissements d'enseignement et de recherche français ou étrangers, des laboratoires publics ou privés. 


\title{
Hierarchical Predictive Control Strategy of microalgae culture in a Photobioreactor
}

\author{
Seif Eddine Benattia, Sihem Tebbani and Didier Dumur \\ Laboratoire des Signaux et Systèmes \\ (L2S, UMR 8506 CentraleSupélec-CNRS-Université Paris-Sud) \\ Control Department, 3 rue Joliot Curie 91192, Gif-sur-Yvette, France \\ Email: \{seifeddine.benattia; sihem.tebbani; didier.dumur\}@ centralesupelec.fr
}

\begin{abstract}
In this paper, the problem of trajectory tracking of a nonlinear system with unknown but bounded model parameters uncertainties is addressed. The proposed control strategy combines a robust model predictive control law with a proportionalintegral (PI) regulator. The predictive controller guarantees the tracking of the reference trajectory, whereas the PI regulator ensures a good tracking accuracy. The proposed robust predictive controller considers only the most influential model parameters (chosen from a sensitivity analysis), and involves the minimization of a regularized optimization problem. This new formulation of the predictive controller ensures a good trade-off between tracking accuracy and computation time. The developed hierarchical strategy is applied to a macroscopic continuous photobioreactor system, for regulating the biomass concentration at a chosen setpoint. Finally, the proposed strategy is validated in simulation to assess its efficiency.
\end{abstract}

Index Terms-Robust predictive control, Min-max optimization problem, Sensitivity analysis, Bioprocesses, Uncertain systems.

\section{INTRODUCTION}

The microalgae cultivation has an increasing interest due to the biochemical characteristics of the microalgae. These micro-organisms are rich in high-value compounds, such as vitamins, pigments, and biologically active compounds. All these compounds can be extracted for use by the cosmetics, pharmaceutical and food industries with more recent applications in sustainable environment, such as wastewater treatment, decomposition of different classes of toxic compounds and carbon capture from industrial flue gases [1]. Biochemical processes are systems where nonlinear effects are significant enough to justify the use of nonlinear models. In the literature of microalgae cultivations, several nonlinear control laws have been developed [2], [3], [4]. They however do not specifically focus on robustness features. In fact, the bioprocess model is uncertain. In addition, the process model is identified and the uncertain parameters are estimated with evaluated confidence intervals. This motivates the development of robust control laws in the presence of modelling uncertainties.

In this work, we propose a robust predictive controller under model parameter uncertainties. The choice of the Model Predictive Control law (MPC) [5] allows the determination of the current control input while taking into account the future system behavior. If the system is uncertain, then the stabilizing properties of the MPC decrease. One of the approaches in the design of robust MPC (RNMPC) [6] controllers is to formulate the problem as a nonlinear min-max optimization problem, where the objective function is minimized for the worst uncertainty realization. The first idea of this study consists in proposing a robust formulation of the NMPC law implying a min-max optimization problem by taking into account only the most influential parameters. The considered parameters are determined on the basis of a sensitivity analysis as proposed in [7]. In addition, in order to reduce as much as possible the computation load, we propose an alternative procedure that transforms the min-max problem into a robust regularized least squares problem [8], through a model linearization technique (first order Taylor series expansion) at each sampling time, along the nominal trajectory [9] (called hereafter reduced Linearized Robust NMPC, rLRMPC). The proposed control strategy is further improved by combining the rLRMPC with a PI control law, similarly to the control structure proposed in [10]. This hierarchical control structure allows reducing the tracking error due to the model approximation through linearization and to the model uncertainties.

The paper is structured as follows. Section 2 presents the class of nonlinear systems that will be considered. In Section 3 , the proposed control strategy is detailed: rLRMPC law, based on the linearization technique combined with the PI controller. An illustrative example (Droop dynamic model of a continuous photobioreactor) is studied in Section 4. Moreover, numerical results are provided to compare different predictive control strategies in case of model mismatch, and highlight the proposed control strategy performances. Finally, some concluding remarks and perspectives are drawn in Section 5.

\section{Problem Statement}

Consider a system described by an uncertain continuous time nonlinear model:

$$
\left\{\begin{array}{l}
\dot{x}(t)=f(x(t), u(t), \theta), \quad x\left(t_{0}=0\right)=x_{0} \\
y(t)=H x(t)
\end{array}\right.
$$

where $x \in \mathbb{R}^{n_{x}}$ is the state vector and $x_{0}$ its initial value, $y \in$ $\mathbb{R}^{n_{y}}$ is the measured output. $f$, of class $C^{1}$ with respect to all its arguments, is the nonlinear process dynamics. $u \in U \subset$ $\mathbb{R}^{n_{u}}$ represents the control input with $U$ the set of admissible controls and $\theta \in \mathbb{R}^{n_{\theta}}$ is the vector of uncertain parameters that are assumed to lie in the admissible region $\Theta=\left[\theta^{-}, \theta^{+}\right]$. The measurement matrix is given by $H \in \mathbb{R}^{n_{y} \times n_{x}}$. Exogenous 
inputs can act on system (1). They are omitted to simplify notation (but are applied to the system).

The control input $u$ is parametrized using a piecewiseconstant approximation $\left(u(\tau)=u\left(t_{k}\right), \tau \in\left[t_{k}, t_{k+1}[)\right.\right.$ over a time interval $\left[t_{k}, t_{k+1}\right] \triangleq\left[k T_{s},(k+1) T_{s}\right]$ considering a constant sampling time $T_{s}$. Let us define the discrete state trajectory $g$ as the solution, at time $t_{k+1}$, of system (1) with initial state $x_{0}$, and with $u_{t_{0}}^{t_{k}}$ the control sequence from the initial time instant $t_{0}$ to the time instant $t_{k}$ :

$$
\begin{cases}x_{k+1}= & g\left(t_{0}, t_{k+1}, x_{0}, u_{t_{0}}^{t_{k}}, \theta\right) \\ y_{k}=H x_{k}\end{cases}
$$

where $x_{k+1}$ is the state at $t_{k+1}, k$ is the time index, $x_{k}$ and $y_{k}$ are the discrete state vector and the sampled measurement at time $t_{k}$, respectively.

This paper aims at designing a control strategy such that the output signal $y_{k}$ tracks the reference signal $\bar{y}_{k}$ while ensuring good closed-loop behaviour and tracking accuracy, despite the model uncertainties and perturbations than can act on the system.

\section{CONTROLlER DESIGN}

\section{A. Hierarchical control strategy}

The proposed control strategy consists in a hierarchical control scheme (Fig. 1), similarly to the one proposed in [10]. The controller is formed by a robust predictive law coupled to a Proportional-Integral (PI) controller. The predictive controller allows tracking the reference trajectory, whereas the PI is added to reduce any residual tracking error. Hereafter, the design of the predictive controller is detailed in Section III-B, and of the PI controller in Section III-C.

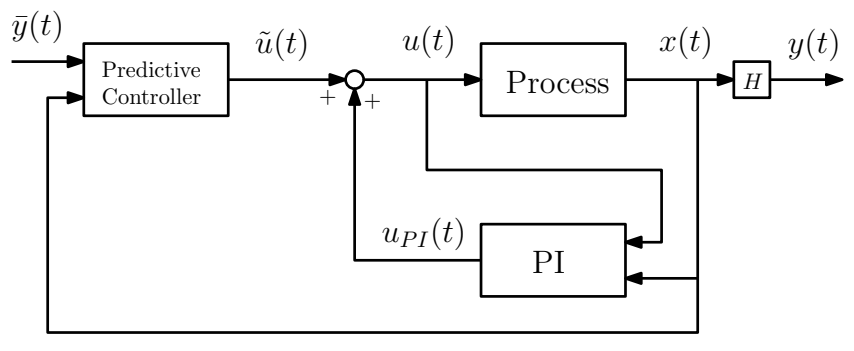

Fig. 1. Scheme of the hierarchical control strategy.

\section{B. Predictive controller design}

1) Robust predictive controller: The predictive controller predicts the plant future evolution over a finite receding horizon of length $N_{p} T_{s}$, using a nonlinear dynamic model. At each time instant $t_{k}$, the future control sequence is computed by minimizing a cost function expressed as a quadratic criterion based on the future tracking errors, while ensuring that all constraints are respected. The first value of this optimal control sequence is applied to the system until the next time step, when the measurement becomes available. The optimization problem is solved again at the next sampling time according to the well-known receding horizon principle.
Since the predictive controller is model-based, it is very sensitive to model uncertainties, and more specifically to the model parameters values. In our case, we will assume that the parameter vector $\theta$ is uncertain and belongs to a known region $\Theta$. In this case, a robust predictive control strategy (RNMPC) implying a min-max optimization problem [6] can be used and expressed as follows (at time index $k$ ):

$$
\tilde{u}_{k}^{k+N_{p}-1}=\arg \min _{u_{k}^{k+N_{p}-1}} \max _{\theta \in \Theta} \Pi\left(u_{k}^{k+N_{p}-1}, \theta\right)
$$

where the cost function is defined as

$$
\begin{aligned}
\Pi\left(u_{k}^{k+N_{p}-1}, \theta\right)= & \left\|u_{k}^{k+N_{p}-1}-\bar{u}_{k}^{k+N_{p}-1}\right\|_{V}^{2}+ \\
& \left\|\hat{y}_{k+1}^{k+N_{p}}-\bar{y}_{k+1}^{k+N_{p}}\right\|_{W}^{2}
\end{aligned}
$$

with $\|z\|_{P}^{2}=z^{\top} P z$ the Euclidean norm weighted by $P$, $u_{k}^{k+N_{p}-1}=\left[u_{k}^{\top}, \ldots, u_{k+N_{p}-1}^{\top}\right]^{\top}$ the optimization variable, $\bar{u}_{k}^{k+N_{p}-1}=\left[u_{k}^{r^{\top}}, \ldots, u_{k+N_{p}-1}^{r^{\top}}\right]^{\top}$ the reference control sequence,

$$
\hat{y}_{k+1}^{k+N_{p}}=\left[\begin{array}{c}
H g\left(t_{k}, t_{k+1}, x_{k}, u_{k}, \theta\right) \\
H g\left(t_{k}, t_{k+2}, x_{k}, u_{k}^{k+1}, \theta\right) \\
\vdots \\
H g\left(t_{k}, t_{k+N_{p}}, x_{k}, u_{k}^{k+N_{p}-1}, \theta\right)
\end{array}\right] \text { the predicted }
$$

output and $\bar{y}_{k+1}^{k+N_{p}}=\left[\bar{y}_{k+1}^{\top}, \ldots, \bar{y}_{k+N_{p}}^{\top}\right]^{\top}$ the reference trajectory. $V \geq 0$ and $W>0$ are tuning weighting matrices (chosen diagonal to simplify the study).

The optimal control sequence $\tilde{u}_{k}^{k+N_{p}-1}$ is determined to minimize the tracking error in the worst case (by considering all trajectories over all possible data scenarii). Since this optimization problem is time consuming, it will be simplified: (i) by reducing the number of the parameters that will be optimized from a sensitivity analysis of the model with respect to its parameters, (ii) and then by linearizing the criterion around the nominal trajectory. These two steps are detailed hereafter.

2) Sensitivity analysis: The sensitivity functions represent the sensitivity of each state $x_{i}$ to (small) variations in each model parameter $\theta_{j}$. Different approaches are possible to determine the sensitivity functions. The most precise method involves analytical derivation [11]. In this case, the dynamics of sensitivities are calculated as follows:

$$
\frac{d}{d t}\left(\frac{\partial x_{i}}{\partial \theta_{j}}\right)=\frac{\partial}{\partial \theta_{j}}\left(\frac{d x_{i}}{d t}\right)=\frac{\partial f_{i}}{\partial \theta_{j}}+\sum_{k=1}^{\operatorname{dim}(x)} \frac{\partial f_{i}}{\partial x_{k}} \frac{\partial x_{k}}{\partial \theta_{j}}
$$

with as an initial condition: $\frac{\partial x_{i}}{\partial \theta_{j}}=0$.

From the analysis of the sensitivity functions temporal evolution, and according to their magnitude order, one can select the parameters which are significantly the most influential on the model [7]. These parameters will be denoted $\kappa$. In the sequel, only the most influential parameters $\kappa$ are considered in the criterion of problem (3), instead of the full model parameters (with $\theta \triangleq[\kappa, \xi]$ ). The other parameters, $\xi$, are set to their nominal values with $\xi_{\text {nom }}=\left(\xi^{+}+\xi^{-}\right) / 2$. 
3) Linearized Robust Model Predictive controller: The min-max optimization problem, even when considering only the most influential parameters, is still time consuming. It will be simplified further by converting the min-max optimization problem into an equivalent minimization one.

From (2), the predicted state for time $t_{k+j}$, starting from state at $t_{k}$, is linearized around the reference trajectory given by the reference control sequence $\bar{u}_{k}^{k+N_{p}-1}$ and for the nominal parameters, $\kappa_{\text {nom }}=\left(\kappa^{+}+\kappa^{-}\right) / 2$. A first order Taylor series expansion of (2) for $j=\overline{1, N_{p}}$ is used:

$$
\begin{aligned}
& g\left(t_{k}, t_{k+j}, x_{k}, u_{k}^{k+j-1},\left[\kappa, \xi_{\text {nom }}\right]\right) \approx g_{\text {nom }}\left(t_{k+j}\right)+ \\
& \nabla_{u} g\left(t_{k+j}\right)\left(u_{k}^{k+j-1}-\bar{u}_{k}^{k+j-1}\right)+\nabla_{\kappa} g\left(t_{k+j}\right)\left(\kappa-\kappa_{\text {nom }}\right)
\end{aligned}
$$

with

$$
\left\{\begin{array}{l|l}
g_{\text {nom }}\left(t_{k+j}\right)=g\left(t_{k}, t_{k+j}, x_{k}, \bar{u}_{k}^{k+j-1},\left[\kappa_{\text {nom }}, \xi_{\text {nom }}\right]\right) \\
\nabla_{\kappa} g\left(t_{k+j}\right)=\frac{\partial g\left(t_{k}, t_{k+j}, x_{k}, u_{k}^{k+j-1},\left[\kappa, \xi_{n o m}\right]\right)}{\partial \kappa} & \begin{array}{l}
u_{k}^{k+j-1}=\bar{u}_{k}^{k+j-1} \\
\kappa=\kappa_{\text {nom }}
\end{array} \\
\nabla_{u} g\left(t_{k+j}\right)=\frac{\partial g\left(t_{k}, t_{k+j}, x_{k}, u_{k}^{k+j-1},\left[\kappa, \xi_{\text {nom }])}\right.\right.}{\partial u_{k}^{k+j-1}} & \begin{array}{l}
u_{k}^{k+j-1}=\bar{u}_{k}^{k+j-1} \\
\kappa=\kappa_{\text {nom }}
\end{array}
\end{array}\right.
$$

The dynamics of sensitivity function with respect to $\kappa$, defined in (9), can be computed for time $t \in\left[t_{k}, t_{k+N_{p}}\right]$ as detailed in (6). In order to simplify the calculation of the gradient $\nabla_{u} g$, finite differences are used to approximate numerically the derivative $\nabla_{u} g\left(t_{k+j}\right)$ for each control $u_{j}, j \in\left[k, k+N_{p}-1\right]$.

From (5) and (7), it comes:

$$
\begin{aligned}
\hat{y}_{k+1}^{k+N_{p}} \approx & \bar{G}_{n o m, k+1}^{k+N_{p}}+\bar{G}_{u, k}^{k+N_{p}-1}\left(u_{k}^{k+N_{p}-1}-\bar{u}_{k}^{k+N_{p}-1}\right) \\
& +\bar{G}_{\kappa, k+1}^{k+N_{p}}\left(\kappa-\kappa_{n o m}\right)
\end{aligned}
$$

with $\left(\bar{G}_{n o m, k+1}^{k+N_{p}}\right)^{T}=\left[H g_{\text {nom }}\left(t_{k+1}\right), \ldots, H g_{\text {nom }}\left(t_{k+N_{p}}\right)\right]$, the vector containing the predicted output for the nominal case,

$\left(\bar{G}_{u, k+1}^{k+N_{p}}\right)^{T}=\left[H \nabla_{u} g\left(t_{k+1}\right), \ldots, H \nabla_{u} g\left(t_{k+N_{p}}\right)\right]$, the vector of Jacobian matrices related to the control sequence,

$\left(\bar{G}_{\kappa, k+1}^{k+N_{p}}\right)^{T}=\left[H \nabla_{\kappa} g\left(t_{k+1}\right), \ldots, H \nabla_{\kappa} g\left(t_{k+N_{p}}\right)\right]$, the vector of Jacobian matrices related to the most influential parameters.

Assuming that the uncertain parameters are uncorrelated, the bounded parametric error can be expressed by:

$$
\begin{gathered}
\kappa-\kappa_{\text {nom }}=\gamma \delta \kappa_{\max } \\
\text { with } \delta \kappa_{\max }=\left(\kappa^{+}-\kappa^{-}\right) / 2 \text { and }\|\gamma\| \leq 1
\end{gathered}
$$

Matrix norm $\|A\|$ is given by $\|A\|=\sqrt{\bar{\sigma}\left(A^{\top} A\right)}$ with $\bar{\sigma}(A)$ the maximum eigenvalue of $\mathrm{A}$.

The min-max optimization problem (3) is converted into a robust regularized least squares problem when applying (1113) in the presence of uncertain data [8], as presented in [9]. Let us consider the following optimization problem:

$$
\min _{z} \max _{\|v\| \leq \pi(z)}\|z\|_{V}^{2}+\|A z-b+C v\|_{W}^{2}
$$

where the perturbation vector $v$ is assumed to satisfy the following factorized form:

$$
C v=C \Delta\left(E_{a} z-E_{b}\right)
$$

where $\Delta$ denotes an arbitrary contraction with $\|\Delta\| \leq 1, C \neq 0$, $E_{a}$ and $E_{b}$ are known quantities of appropriate dimensions. The nonnegative function $\pi(z)$ is assumed to be a known bound on the perturbation $v$ and is a function of $z$ only, given by:

$$
\pi(z)=\left\|E_{a} z-E_{b}\right\|
$$

Introducing the Lagrange multiplier $\lambda$, the problem (14) becomes equivalent to [8]:

$$
\min _{\lambda \geq\left\|C^{\top} W C\right\|} \min _{z} z^{\top} V z+(A z-b)^{\top} W(\lambda)(A z-b)+\lambda \pi(z)^{2}
$$

where the minimizer $z$ must satisfy the equation

$$
z(\lambda)=E(\lambda)^{-1}\left(A^{\top} W(\lambda) b+\lambda E_{a}^{\top} E_{b}\right)
$$

with

$$
E(\lambda)=V(\lambda)+A^{\top} W(\lambda) A+\lambda E_{a}^{\top} E_{a}
$$

The modified weighting matrices $V(\lambda)$ and $W(\lambda)$ are obtained from $V$ and $W$ via:

$$
\left\{\begin{array}{l}
V(\lambda)=V+\lambda E_{a}^{\top} E_{a} \\
W(\lambda)=W+W C\left(\lambda I-C^{\top} W C\right)^{\dagger} C^{\top} W
\end{array}\right.
$$

The invertibility of $E(\lambda)$ is guaranteed by the positive definiteness of $V$.

The nonnegative scalar parameter $\lambda^{0} \in \mathbb{R}$ solution of (17), is computed from the following unidimensional minimization problem:

$$
\begin{array}{ll}
\lambda^{\circ}=\arg \min _{\lambda \geq\left\|C^{\top} W C\right\|} & \|z(\lambda)\|_{V}^{2}+\lambda\left\|E_{a} z(\lambda)-E_{b}\right\|^{2}+ \\
& \|A z(\lambda)-b\|_{W(\lambda)}^{2}
\end{array}
$$

Finally, the problem has a unique global minimum $z^{\mathrm{o}}$ given by (18) for $\lambda=\lambda^{\circ}$.

Based on this formalism, the robust nonlinear predictive problem which is defined by (3-4), is written in the form (14-16) with:

$$
\left\{\begin{array}{l}
z=u_{k}^{k+N_{p}-1}-\bar{u}_{k}^{k+N_{p}-1}, \\
A=\bar{G}_{u, k}^{k+N_{p}-1}, b=\bar{y}_{k+1}^{k+N_{p}}-\bar{G}_{n o m, k+1}^{k+N_{p}}, \\
C=\bar{G}_{\kappa, k+1}^{k+N_{p}}, \Delta=\gamma, E_{a}=0, E_{b}=-\delta \kappa_{\text {max }}
\end{array}\right.
$$

The application of (18-21) provides the solution of (3-4) as follows [9]:

step 1. $\lambda^{0}$ is computed from the following minimization problem:

$$
\lambda^{\mathrm{o}}=\arg \min _{\lambda \geq \| \bar{G}_{\kappa, k+1}^{k+N_{p}^{\top} W \bar{G}_{\kappa, k+1}^{k+N_{p}} \|}} G(\lambda)
$$

where the function $G(\lambda)$ is defined by:

$$
\begin{aligned}
G(\lambda)= & \left\|\bar{G}_{u, k}^{k+N_{p}-1} z(\lambda)-\bar{y}_{k+1}^{k+N_{p}}+\bar{G}_{\text {nom }, k+1}^{k+N_{p}}\right\|_{W(\lambda)}^{2}+ \\
& \|z(\lambda)\|_{V}^{2}+\lambda\left\|\delta \kappa_{\max }\right\|^{2}
\end{aligned}
$$




$$
\text { with } \begin{aligned}
z(\lambda)= & {\left[V+\bar{G}_{u, k}^{k+N_{p}-1^{\top}} W(\lambda) \bar{G}_{u, k}^{k+N_{p}-1}\right]^{-1} } \\
& {\left[\bar{G}_{u, k}^{k+N_{p}-1^{\top}} W(\lambda)\left(\bar{y}_{k+1}^{k+N_{p}}-\bar{G}_{\text {nom }, k+1}^{k+N_{p}}\right)\right] }
\end{aligned}
$$

and

$$
W(\lambda)=W+W \bar{G}_{\kappa, k+1}^{k+N_{p}}\left(\lambda I-\bar{G}_{\kappa, k+1}^{k+N_{p}^{\top}} W \bar{G}_{\kappa, k+1}^{k+N_{p}}\right)^{\dagger} \bar{G}_{\kappa, k+1}^{k+N_{p}^{\top}} W
$$

step 2. The control sequence is derived from (18):

$$
\begin{aligned}
\tilde{u}_{k}^{k+N_{p}-1}= & \bar{u}_{k}^{k+N_{p}-1}+\left[V+\bar{G}_{u, k}^{k+N_{p}-1^{\top}} W\left(\lambda^{\mathrm{o}}\right) \bar{G}_{u, k}^{k+N_{p}-1}\right]^{-1} \\
& {\left[\bar{G}_{u, k}^{k+N_{p}-1^{\top}} W\left(\lambda^{\mathrm{o}}\right)\left(\bar{y}_{k+1}^{k+N_{p}}-\bar{G}_{\text {nom }, k+1}^{k+N_{p}}\right)\right] }
\end{aligned}
$$

with $W\left(\lambda^{\circ}\right)$ given in (26) for $\lambda=\lambda^{\circ}$.

The minimum $\lambda^{\circ}$ of the unidimensional function $G(\lambda)$ is found using the golden section search algorithm.

As a conclusion, the predictive controller consists in solving online an unidimensional optimization problem (23-24) at each sampling time, instead of solving min-max problem (3-4). In the sequel, this predictive control law will be referred to as reduced linearized robust model predictive controller (rLRMPC).

\section{PI controller design}

At each time instant $t_{k}=k T_{s}$, the optimal control law $\tilde{u}(t)$ obtained from the predictive controller (27) is completed by a Proportional-Integral law $u_{P I}(t)$. The idea is to drive the system to track the predicted output $\hat{y}$ in order to cancel the difference between the model prediction output and the system output. In this study, the PI design is performed in continuoustime, then discretized for implementation. Let us define the model prediction resulting from the application of the previous control input for $t \in\left[t_{k-1}, t_{k}\right]$, as follows:

$$
\hat{y}(t)=H g\left(t_{k-1}, t, x_{k-1}, u_{k-1}, \theta_{\text {nom }}\right)
$$

In the case of the considered control problem, the control law derived from the PI strategy is given by (with $K_{p}$ and $T_{i}$ the controller parameters):

$$
u_{P I}(t)=K_{p}(y(t)-\hat{y}(t))+\frac{K_{p}}{T_{i}} \int_{t_{k-1}}^{t}(y(\tau)-\hat{y}(\tau)) d \tau
$$

Finally, with (29) evaluated at $t=t_{k}$, the control input to be applied to the plant is obtained as the sum of two parts, given by:

$$
u\left(t_{k}\right)=\tilde{u}\left(t_{k}\right)+u_{P I}\left(t_{k}\right)
$$

The component $\tilde{u}\left(t_{k}\right)$ (the first value of the optimal control sequence) is generated by the rLRMPC controller, while $u_{P I}\left(t_{k}\right)$ is generated by the PI controller.

\section{ILLUSTRATIVE EXAMPLE}

The process under consideration here is a continuous photobioreactor, without any additional biomass in the feed, and neglecting the effect of gas exchanges. Thus, the dynamic equations resulting from mass balances are given by [2]:

$$
\left\{\begin{array}{c}
\dot{X}(t)=\mu(Q(t), I(t)) X(t)-D X(t) \\
\dot{Q}(t)=\rho(S(t))-\mu(Q(t), I(t)) Q(t) \\
\dot{S}(t)=\left(S_{\text {in }}-S(t)\right) D-\rho(S(t)) X(t)
\end{array}\right.
$$

where $D$ represents the dilution rate $\left(\mathrm{d}^{-1}, \mathrm{~d}\right.$ : day), $X$ the biomass concentration $\left(\mu \mathrm{m}^{3} \mathrm{~L}^{-1}\right), Q$ the internal quota $\left(\mu \mathrm{mol} \mu \mathrm{m}^{-3}\right), S$ the substrate concentration $\left(\mu \mathrm{mol} \mathrm{L}{ }^{-1}\right)$ and $I$ the light intensity $\left(\mu \mathrm{E} \mathrm{m}^{-2} \mathrm{~s}^{-1}\right)$.

The specific uptake rate, $\rho(S)$, and the specific growth rate, $\mu(Q, I)$, are given by:

$$
\rho(S)=\rho_{m} \frac{S}{S+K_{S}} \text { and } \mu(Q, I)=\bar{\mu} \frac{1-K_{Q} / Q}{I+K_{S I}+I^{2} / K_{i I}}
$$

The nonlinear model is represented in the state-space formal-

\begin{tabular}{|c|c|c|}
\hline Parameter & Value & Unit \\
\hline $\bar{\mu}$ : maximal specific growth rate & 2 & $\mathrm{~d}^{-1}$ \\
\hline$\rho_{m}$ : maximal specific uptake rate & 9.3 & $\mu \mathrm{mol} \mu \mathrm{m}^{-3} \mathrm{~d}^{-1}$ \\
\hline$K_{Q}:$ minimal cell quota & 1.8 & $\mu \mathrm{mol} \mu \mathrm{m}^{-3}$ \\
\hline$K_{s}$ : substrate half saturation constant & 0.105 & $\mu \mathrm{mol} \mathrm{L}{ }^{-1}$ \\
\hline$K_{S I}:$ light saturation constant & 150 & $\mu \mathrm{E} \mathrm{m}^{-2} \mathrm{~s}^{-1}$ \\
\hline$K_{i I}$ : light inhibition constant & 2000 & $\mu \mathrm{E} \mathrm{m}^{-2} \mathrm{~s}^{-1}$ \\
\hline$S_{\text {in }}$ : inlet substrate concentration & 100 & $\mu \mathrm{mol} \mathrm{L}{ }^{-1}$ \\
\hline$I_{o p t}=\sqrt{K_{s I} K_{i I}}:$ optimal light intensity & 547 & $\mu \mathrm{E} \mathrm{m}^{-2} \mathrm{~s}^{-1}$ \\
\hline
\end{tabular}
ism (1), with:

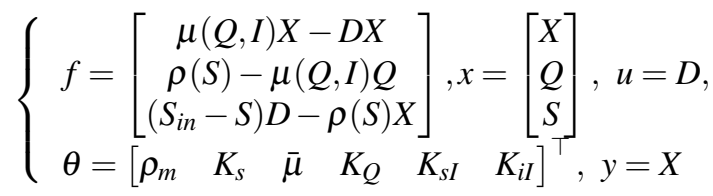

To simplify notations, the exogenous inputs $\left(S_{i n}, I\right)$ are omitted but are applied. The model parameters are displayed in Table I [12], [13]. Details related to the modelling can be found in [14].

TABLE I

MODEL PARAMETERS.

The main objective of the control is to regulate the biomass concentration $X$ to a reference value $X^{r}$, while the dilution rate $D$ is constrained to track the reference $D^{r}$. The latter is computed from the knowledge of the targeted setpoint at each time instant as detailed in [7]. According to results obtained in [7], the substrate half saturation constant $K_{S}$ and the minimal cell quota $K_{Q}$ are the most influential parameters on the biomass concentration evolution $\left(\kappa=\left[K_{s}, K_{Q}\right]\right)$.

Now, the efficiency of the proposed control strategy is validated in simulation. The performances of the above mentioned algorithms are compared in a worst uncertain parameters case. The parameters values of the system are chosen on the parameter subspace border $\left(\theta_{\text {real }}=\left[\rho_{m}^{+}, K_{s}^{-}, \bar{\mu}^{+}, K_{Q}^{-}, K_{s I}^{-}, K_{i I}^{+}\right]\right)$ [7], where the uncertain parameters subspace $\left[\theta^{-}, \theta^{+}\right]$is given by $\left[0.8 \theta_{\text {nom }}, 1.2 \theta_{\text {nom }}\right]$. The maximal admissible dilution rate $D_{\max }$ equals $1.6 d^{-1}$. The simulation time $T_{f}$ and the sampling time $T_{S}$ are chosen equal to 1 day and $20 \mathrm{~min}$ respectively. The inlet substrate concentration $S_{i n}$ is assumed to be perfectly known. The controllers tuning parameters are determined by a trial-and-error technique: $N_{p}=5, V=I_{5}$ and $W=I_{5}$ for the predictive strategy and $K_{p}=1$ and $T_{i}=0.01 d^{-1}$ for the PI controller. Simulations have been carried out considering the uncertain parameter worst case cited previously. 
First, the light intensity is assumed to be measured and constant, equal to $I_{o p t}$ (that maximises $\mu(Q, I)$ [11]). Biomass concentration measurements, $y_{k}$, are assumed to be corrupted by a Gaussian white noise of zero mean and 0.1 standard deviation. The response of the system for a rising edge is studied. The obtained results are depicted in Fig. 2. This figure compares the (r)LRMPC and (r)LRMPC-PI controllers performances (LRMPC is similar as in Section III-B3 when considering all the parameters in the optimization step, i.e. no reduction in the number of the parameters).
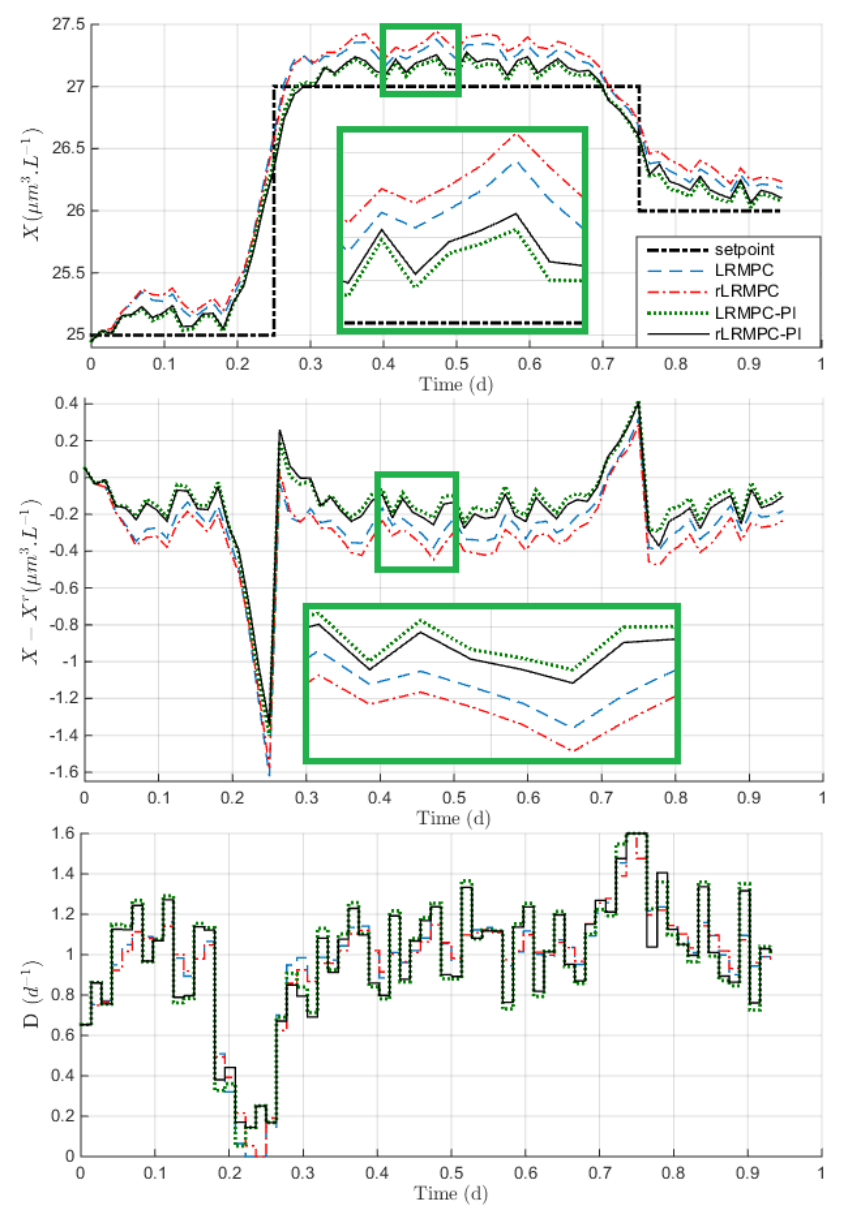

Fig. 2. Biomass concentration and dilution rate evolution with time for (r)LRMPC and (r)LRMPC-PI strategies.
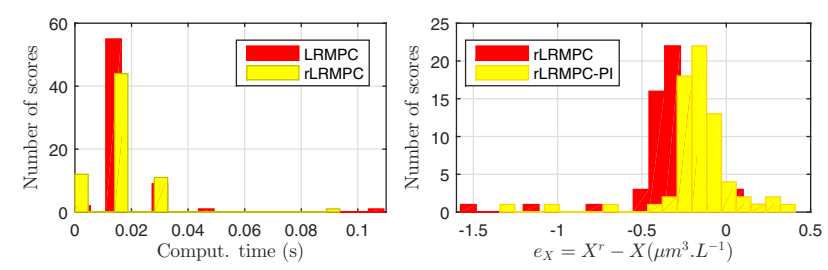

Fig. 3. Histogram of the distribution of the computation time (left) and the biomass concentration tracking error (right).

It can be noticed the anticipation of a setpoint change due to the prediction of the model behavior in the moving horizon.
In the case of (r)LRMPC law, a static error is present, due to the superposition of two phenomena: the approximation of the model through linearization, and the model error. On the other hand, the use of a hierarchical scheme (see Fig. 1) for both the two (r)LRMPC-PI laws allows reducing the static error (45\% in comparison with (r)LRMPC laws as shown in Fig. 3). Moreover, considering only the most influential parameters (rLRMPC, with and without PI) leads to a decrease of the online computation load (about $15 \%$ in comparison with LRMPC as shown in Fig. 3) while keeping an acceptable level of the tracking accuracy.

Nevertheless, the tracking error is not completely cancelled with this choice of $T_{i}$. It could be reduced by decreasing the $T_{i}$ value, but inducing a more fluctuant control signal. A compromise must thus be found.

Secondly, a day/night light variation is considered, modeled as [2]: $I(t)=I_{\text {opt }}(\max \{0, \sin (2 \pi t)\})^{2}$, where the time $t$ is in days. The light intensity modeling is assumed mismatched (20\% as shown in Fig.4) and used in the prediction step. The biomass concentration setpoint is constant $\left(X^{r}=25 \mu \mathrm{m}^{3} / L\right)$. Figure 4 illustrates the performances of the proposed strategy and compares it to those of a more classical Robust NMPC as defined in (3).
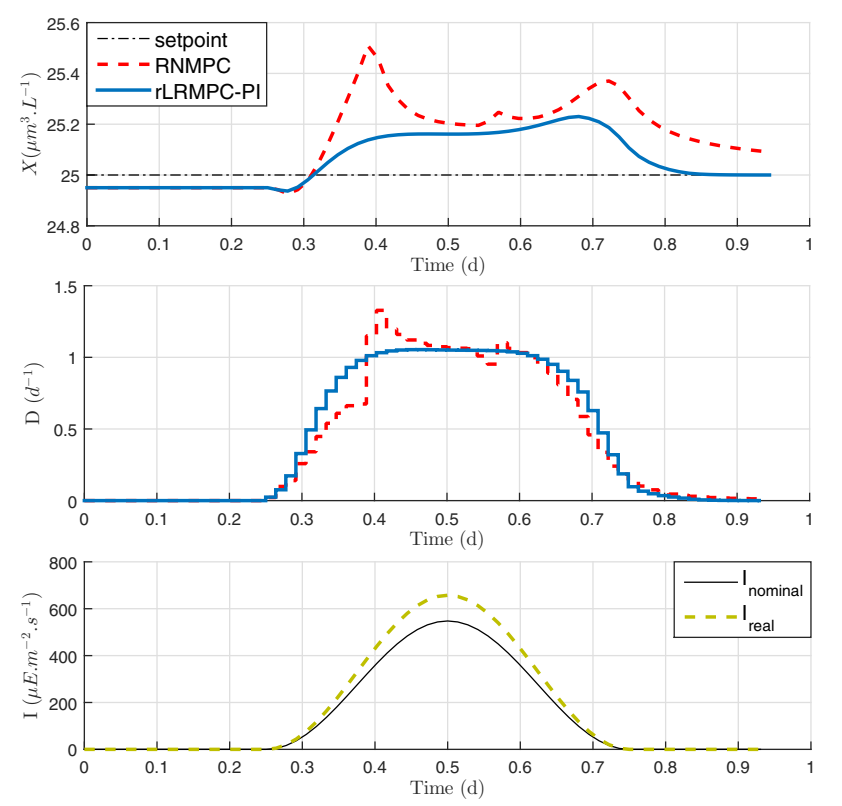

Fig. 4. Temporal evolutions of biomass concentration tracking error, dilution rate and light intensity.

TABLE II

COMPARISON OF THE COMPUTATION TIMES

\begin{tabular}{|c|c|c|c|}
\cline { 2 - 4 } \multicolumn{1}{c|}{} & \multicolumn{3}{c|}{ Computation time (s) } \\
\hline Controller & min & mean & max \\
\hline rLRMPC-PI & $<10^{-5}$ & 0.014 & 0.08 \\
RNMPC & 0.125 & 3.73 & 17.64 \\
\hline
\end{tabular}

Both strategies counter the effect of fluctuations in light intensity. It can be observed that the proposed approach (rLRMPC-PI) reduces the fluctuations of biomass concentration better than the RNMPC, especially at the end of the 
culture duration. The computation times are given in Table II. It can be noticed the reduction of the computation time with the proposed strategy in comparison to the RNMPC law. In conclusion, the proposed approach is robust against parameter uncertainties and less sensitive to light variations than the RNMPC.

\section{CONCLUSION}

In this paper, we have proposed a hierarchical control strategy for a nonlinear system with bounded model uncertainties. The developed approach is formed by a two-level controller: a reduced linearized robust MPC and a PI control law. The firstorder linearization of the criterion of the Robust NMPC law, in combination to the reduction of the number of uncertain parameters, reduces the computation load while assuring a good tracking behavior. The linearized robust MPC is further combined with a classical Proportional-Integral controller to improve the accuracy of the reference tracking. Work under progress considers the proof of the stability of the proposed control scheme, and future work could consider a robust NMPC in case of unmodeled uncertainties and an experimental validation.

\section{REFERENCES}

[1] P. Spolaore, C. Joannis-Cassan, E. Duran, and A. Isambert, "Commercial applications of microalgae," J Biosci. Bioeng., vol. 101, pp. 87-96, 2006.

[2] P. Masci, F. Grognard, and O. Bernard, "Microalgal biomass surface productivity optimization based on a photobioreactor model," Proc. of the 11th CAB Conference, 2010, leuven, Belgium.

[3] G. A. Ifrim, M. Titica, M. Barbu, L. Boillereaux, G. Cogne, S. Caraman, and J. Legrand, "Multivariable feedback linearizing control of chlamydomonas reinhardtii photoautotrophic growth process in a torus photobioreactor," Chem. Eng. J., vol. 218, pp. 191-203, 2013.

[4] S. Tebbani, F. Lopes, R. Filali, D. Dumur, and D. Pareau, "Nonlinear predictive control for maximization of $\mathrm{CO}_{2}$ bio-fixation by microalgae in a photobioreactor," Bioprocess Biosyst. Eng., vol. 37, pp. 83-97, 2014.

[5] E. F. Camacho and C. Bordons, Model Predictive Control. Springer London, 2004.

[6] E. C. Kerrigan and J. Maciejowski, "Feedback min-max model predictive control using a single linear program: Robust stability and the explicit solution," Int. J. Robust Nonlinear Control, vol. 14, pp. 395-413, 2004.

[7] S. E. Benattia, S. Tebbani, D. Dumur, and D. Selisteanu, "Robust nonlinear model predictive controller based on sensitivity analysis application to a continuous photobioreactor," Proc. of the 2014 IEEE $M S C$, pp. 1705-1710, 2014, antibes/Nice, France.

[8] A. H. Sayed, V. H. Nascimento, and F. A. M. Cipparrone, "A regularized robust design criterion for uncertain data," SIAM J. MAT. ANAL. APPL. vol. 32:4, pp. 1120-1142, 2002.

[9] S. E. Benattia, S. Tebbani, and D. Dumur, "Robust nonlinear model predictive control for regulation of microalgae culture in a continuous photobioreactor," Proc. of the 1st Conference on Modelling Identification and Control of Nonlinear Systems, pp. 202-207, 2015, st. Petersburg,Russia.

[10] M. Rubagotti, D. M. Raimondo, A. Ferrara, and L. Magni, "Robust model predictive control of continuous-time sampled data nonlinear systems with interval sliding mode," IEEE Transaction on Automatic Control, vol. 56, no. 3, pp. 556-570, 2011.

[11] D. Dochain, Automatic control of bioprocesses, D. Dochain, Ed. Editor. John Wiley \& Sons, 2008.

[12] G. Goffaux and A. Vande Wouwer, "Design of a robust nonlinear receding-horizon observer-Application to a biological system," Proc. of the 17th IFAC World Congress, pp. 15 553-15 558, 2008, seoul, Korea.

[13] R. Munoz-Tamayo, P. Martinon, G. Bougaran, F. Mairet, and O. Bernard, "Getting the most out of it: optimal experiments for parameter estimation of microalgae growth models," J. of Process Control, vol. 24, no. 6, pp. 991-1001, 2014
[14] S. E. Benattia, S. Tebbani, and D. Dumur, "Nonlinear model predictive control for regulation of microalgae culture in a continuous photobioreactor," Proc. of the 22nd MED Conference, pp. 469-474, 2014, palermo, Italy. 